\title{
ArcheoSciences
}

Revue d'archéométrie

41-2 | 2017

Varia

\section{Chronologie et mutations fonctionnelles dans la poterie-tuilerie médiévale de la Goulande (La Haute-Chapelle, Orne)}

Avec une nouvelle datation du site de Saint-Georges-de-Rouelley

(Manche)

Chronology and functional mutations in the medieval pottery-tilery workshop of la Goulande (La Haute-Chapelle, Orne). With a new dating of pottery workshop of Saint-Georges-de-Rouelley (Manche)

Bruno Fajal, Yves Gallet, Philippe Lanos et Maxime Le Goff

Traducteur : Philippe Bernouis

\section{OpenEdition}

Journals

Édition électronique

URL : https://journals.openedition.org/archeosciences/4988

DOI : 10.4000/archeosciences.4988

ISBN : 978-2-7535-7393-2

ISSN : 2104-3728

Éditeur

Presses universitaires de Rennes

Édition imprimée

Date de publication : 8 décembre 2017

Pagination : 53-61

ISBN : 978-2-7535-7391-8

ISSN : 1960-1360

Référence électronique

Bruno Fajal, Yves Gallet, Philippe Lanos et Maxime Le Goff, « Chronologie et mutations fonctionnelles dans la poterie-tuilerie médiévale de la Goulande (La Haute-Chapelle, Orne) », ArcheoSciences [En ligne], 41-2 | 2017, mis en ligne le 08 décembre 2019, consulté le 20 juillet 2021. URL : http:// journals.openedition.org/archeosciences/4988; DOI : https://doi.org/10.4000/archeosciences.4988 


\title{
Chronologie et mutations fonctionnelles dans la poterie-tuilerie médiévale de la Goulande (La Haute-Chapelle, Orne). Avec une nouvelle datation du site de Saint-Georges- de-Rouelley (Manche)
}

\author{
Chronology and Functional Mutations in the Medieval Pottery-tilery Workshop \\ of la Goulande (La Haute-Chapelle, Orne). With a New Dating of Pottery Workshop \\ of Saint-Georges-de-Rouelley (Manche)
}

\author{
Bruno Fajal $^{a}$, Yves Gallet ${ }^{b}$ et Philippe Lanos $^{c}$ \\ avec la collaboration de Maxime Le Goff ${ }^{\mathrm{d}}$, Philippe Bernouis ${ }^{\mathrm{e}}$ \\ et Michael BATT ${ }^{\mathrm{f}}$
}

\begin{abstract}
Résumé : L'atelier de la Goulande (La Haute-Chapelle, Orne) se trouve au sein d'un vaste ensemble de fosses d'extraction d'argiles utilisées pour fabriquer de la poterie commune, des grès et des tuiles. Les prospections géophysiques menées à cet endroit ont permis de distinguer deux anomalies magnétiques, correspondant à trois fours référencés $\mathrm{A}, \mathrm{B}$ et $\mathrm{C}$. Le premier $(\mathrm{A})$ est un four à pots longitudinal, daté par une analyse archéomagnétique de la seconde moitié du XIII ${ }^{\mathrm{e}}$ siècle ou du début du XIV e siècle. Sa morphologie et les productions qu'on peut lui associer sont proches de celles de l'atelier voisin de Saint-Georges-de-Rouelley, dont le réexamen des données archéomagnétiques recueillies au milieu des années 1980 conduit à une datation plus haute d'un demi-siècle (1260-1280 au lieu de 1325-1350). Les deux ateliers de potiers (Saint-Georges-de-Rouelley et four A de la Goulande) seraient donc sensiblement contemporains. À environ 1,50 m plus à l'est, se trouvent les restes des deux autres fours. Le premier (B) est un four à tuiles, auquel s'est substitué un four à pots longitudinal (C). En accord avec les données archéologiques, les résultats archéomagnétiques obtenus donnent des datations différentes pour chacun des fours étudiés. La production de tuiles qui s’y est développée a probablement pris fin au début du $\mathrm{Xv}^{\mathrm{e}}$ siècle. Finalement, une production de grès s'est substituée à la production de tuiles, vraisemblablement jusqu'au dernier tiers du $\mathrm{Xv}^{\mathrm{e}}$ siècle.
\end{abstract}

Abstract: The workshop of la Goulande (La Haute-Chapelle, Orne) is located close to a large complex of pits used to extract clay to make earthenware pottery, stonewares and tiles. Geophysical surveys have identified two magnetic anomalies corresponding to three kilns referenced $A$, $B$, and $C$. The first (A) is a longitudinal pottery kiln, dated by archaeomagnetic analysis to the second half of the $13^{\text {th }}$ century or to the beginning of the $14^{\text {th }}$ century. Its morphology and productions are similar to those of the neighbouring workshop of Saint-Georges-de-Rouelley, for which the re-examination of the

${ }^{a}$ CNRS, CRAHAM-UMR 6273, Université de Normandie, Unicaen. (bruno.fajal@unicaen.fr)

${ }^{b}$ CNRS, IPGP, 1, rue Jussieu, 75238 PARIS cedex 05. (gallet@ipgp.fr)

' CNRS, IRAMAT-CRP2A, UMR 5060, Université de Bordeaux-Montaigne et Géosciences-Rennes, UMR 6118, Université de Rennes 1. (philippe. lanos@univ-rennes1.fr)

${ }^{d}$ CNRS, IPGP, 1, rue Jussieu, 75238 PARIS cedex 05. (legoff@ipgp.fr)

'Conseil général du Calvados, Service du Patrimoine, 36, rue Fred-Scamaroni, 14000 CAEN. (philippe.bernouis@calvados.fr)

${ }^{f}$ Membre associé, CRAHAM-UMR 6273, Université de Normandie, Unicaen. (michael.batt@wanadoo.fr) 
archaeomagnetic data collected during the 1980s provides us with a date earlier by half a century (1260-1280 instead of 1325-1350). The two potters workshops (Saint-Georges-de-Rouelley and kiln A at la Goulande) are therefore contemporary. Around 1,50 m to the east, the remains of two other kilns were found. The first (B), a tile kiln, was replaced by a longitudinal pottery kiln (C). In correspondence with the archaeological data, archaeomagnetic results obtained provide different dates for each of the kilns studied. Finally, the production of stoneware most probably substituted the production of tiles up until the last third of the $15^{\text {th }}$ century.

Mots clés : Normandie, four à pots, four à tuiles, poteries communes, grès, XIII ${ }^{\mathrm{e}}$ siècle, XIV ${ }^{\mathrm{e}}$ siècle, $\mathrm{XV}^{\mathrm{e}}$ siècle, archéomagnétisme, la Goulande, Saint-Georges-de-Rouelley

Keywords: Normandy, pottery kiln, tile kiln, earthenware, stoneware, $13^{\text {th }}$ century, $14^{\text {th }}$ century, $15^{\text {th }}$ century, archaeomagnetism, la Goulande, SaintGeorges-de-Rouelley

À la fin des années 1980, un programme de recherche sur l'apparition et le développement de l'artisanat de la terre cuite et du grès céramique en Normandie méridionale a

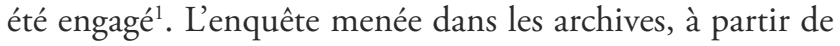
la microtoponymie et sur le terrain, autour des gisements incluant les argiles grésantes à l'origine des productions de grès domfrontais (Bucur et al., 1989) a révélé une vingtaine d'ateliers céramiques, médiévaux et/ou modernes. L'un d'eux, présumé médiéval, a été localisé près du hameau de la Goulande, dans un herbage, sous les racines d'un vieux poirier couché lors de la violente tempête qui traversa la Normandie le 15 octobre 1987.

\section{LE CONTEXTE DE L'ÉTUde}

Dans un article récent (Fajal, Marguerie et al., 2015), nous rappelions les raisons pour lesquelles le CRAHAM avait engagé dans les années 1980 une vaste enquête sur l'artisanat de la terre cuite et du grès en Normandie méridionale, dans les régions de Domfront et de Mortain. Il fallait, en premier lieu, répondre à une demande croissante, régionale et étrangère, d'informations sur l'origine de fabrication de grès réputés normands. Il s'agissait d'en définir l'ampleur, sur le terrain, d'en préciser la chronologie et les conditions de développement. Il s'agissait enfin d'étudier les vestiges d'ateliers, fours et produits céramiques associés, à une période où nos connaissances sur ce type de vestiges étaient encore très lacunaires.

Lors de l'enquête de terrain, nous avions localisé des vestiges d'ateliers de potiers mais aussi de tuiliers, ainsi que des

1. Enquête réalisée dans le cadre d'un programme de prospections et d'opérations archéologiques sur des ateliers producteurs de grès médiévaux et post-médiévaux de la région de Domfront, en Basse-Normandie (coord. B. Fajal). $\mathrm{N}^{\circ}$ de site : 61.201.001 AH. Financements DRAC de Basse-Normandie et CRA[HA]M. L'article reprend les données présentées dans une communication-poster au colloque du GMPCA (Caen, 2013). ateliers qui présentaient les traces des deux activités potière et tuilière (Dufournier et Fajal, 1995). L'un d'eux a été localisé aux confins des communes de Saint-Gilles-des-Marais (où se trouve le hameau de la Goulande, éponyme pour les carrières d'argile), et de La Haute-Chapelle, où se trouve l'installation artisanale médiévale.

La poterie-tuilerie de la Goulande est située à environ trois kilomètres à l'ouest de la ville de Domfront (figure 1a) ${ }^{2}$. L'atelier borde un vaste ensemble de fosses d'extraction d'argiles kaolinitiques, occupant un petit fossé tectonique de quelques $\mathrm{km}^{2}$, au nord du confluent des rivières Égrenne et Varenne (Lautridou, 2002; figure 1b). Ces fosses prennent aujourd'hui la forme de mares, encore bien visibles sur les cartes IGN des années $1980^{3}$. Les argiles extraites de ces carrières ont servi aux artisans de la région pour fabriquer des poteries et des terres cuites architecturales (Mauger, 1905). De couleur gris-clair, très plastique, l'une de ces argiles, aux propriétés grésantes, a été utilisée pour mettre au point puis pour commencer à produire, avant le tournant du XIv siècle, les premiers grès normands (Bucur et al., 1989, complété par une note sur l'atelier de Saint-Georges-de-Rouelley, ciaprès). Les prospections géophysiques réalisées à cet endroit ont distingué deux anomalies magnétiques, très proches l'une de l'autre, correspondant aux vestiges de trois fours dont deux - un four à tuiles et un four à pots - occupent le même emplacement.

2. Coord. X : 48.59474; Y : $-0.688577 ; Z: 124,50 \mathrm{~m}$.

3. Carte IGN Domfront 1-2, feuille XV-16, Institut géographique national, 1975; carte IGN 1516 O, série bleue, 1988 (2éd.), autour des hameaux du Bois de Landelle, de Launay, du Fresne et de la Goulande [C IGN 2016, autorisation 43-16030, reproduction interdite]. Une fiche réalisée par l'Inventaire du patrimoine géologique de Basse-Normandie sous le titre " Argiles plio-pléistocènes de Saint-Gilles-des-Marais » livre une description géologique sommaire et quelques photos récentes de certaines de ces fosses remplies d'eau. http://www.apgn.frlapgn/carte_sitel fic_s61_19.pdf. 
Figure 1a : La

Haute-Chapelle, le Domfrontais et les zones de production de grès en Normandie.

Figure 1a: La Haute-Chapelle, the Domfrontais and stoneware production sites in Normandy.
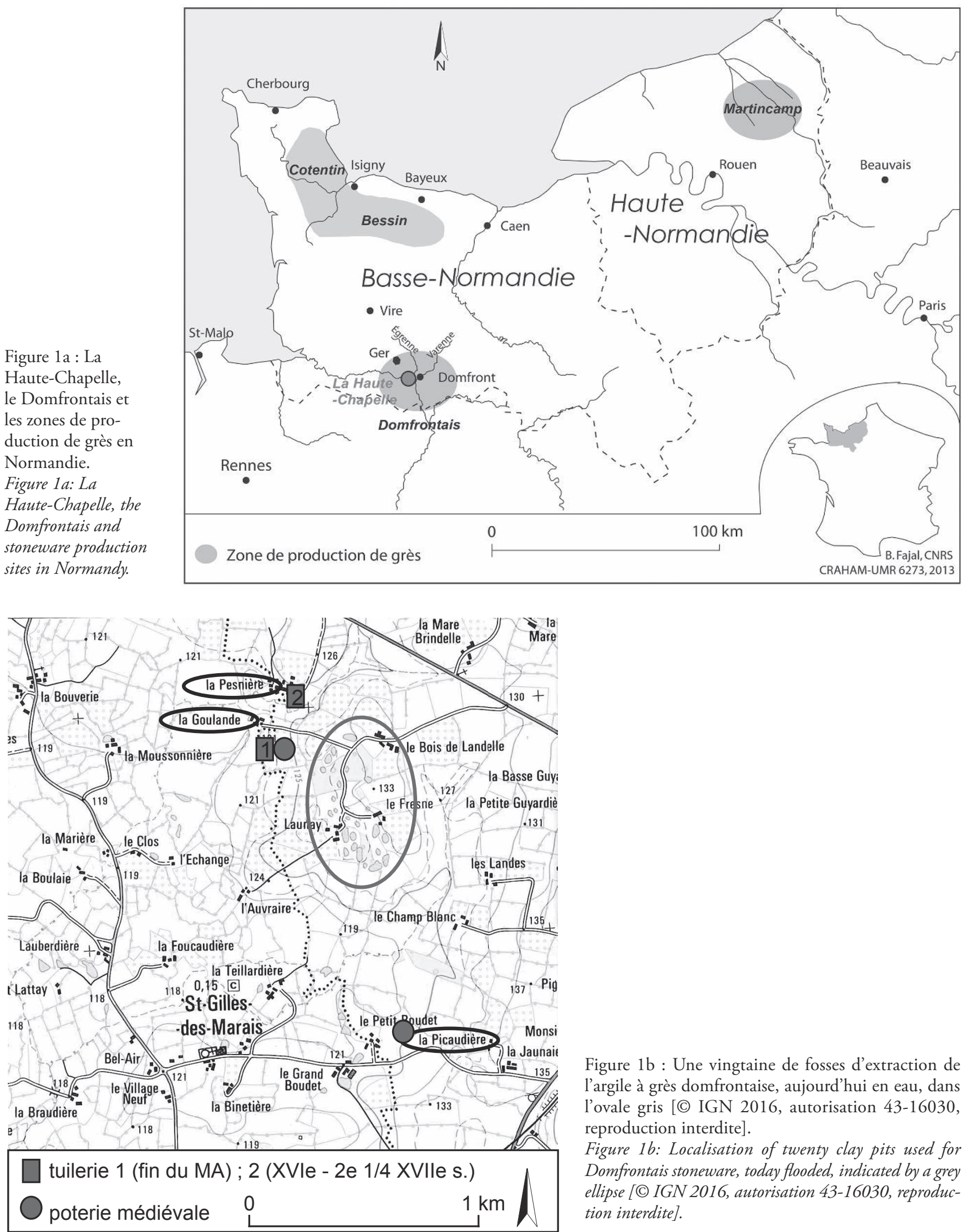

Figure $1 \mathrm{~b}$ : Une vingtaine de fosses d'extraction de l'argile à grès domfrontaise, aujourd'hui en eau, dans l'ovale gris [@ IGN 2016, autorisation 43-16030, reproduction interdite].

Figure 1b: Localisation of twenty clay pits used for Domfrontais stoneware, today flooded, indicated by a grey ellipse [C IGN 2016, autorisation 43-16030, reproduction interdite]. 


\section{2. ÉTUdE ARCHÉOLOGIQUE}

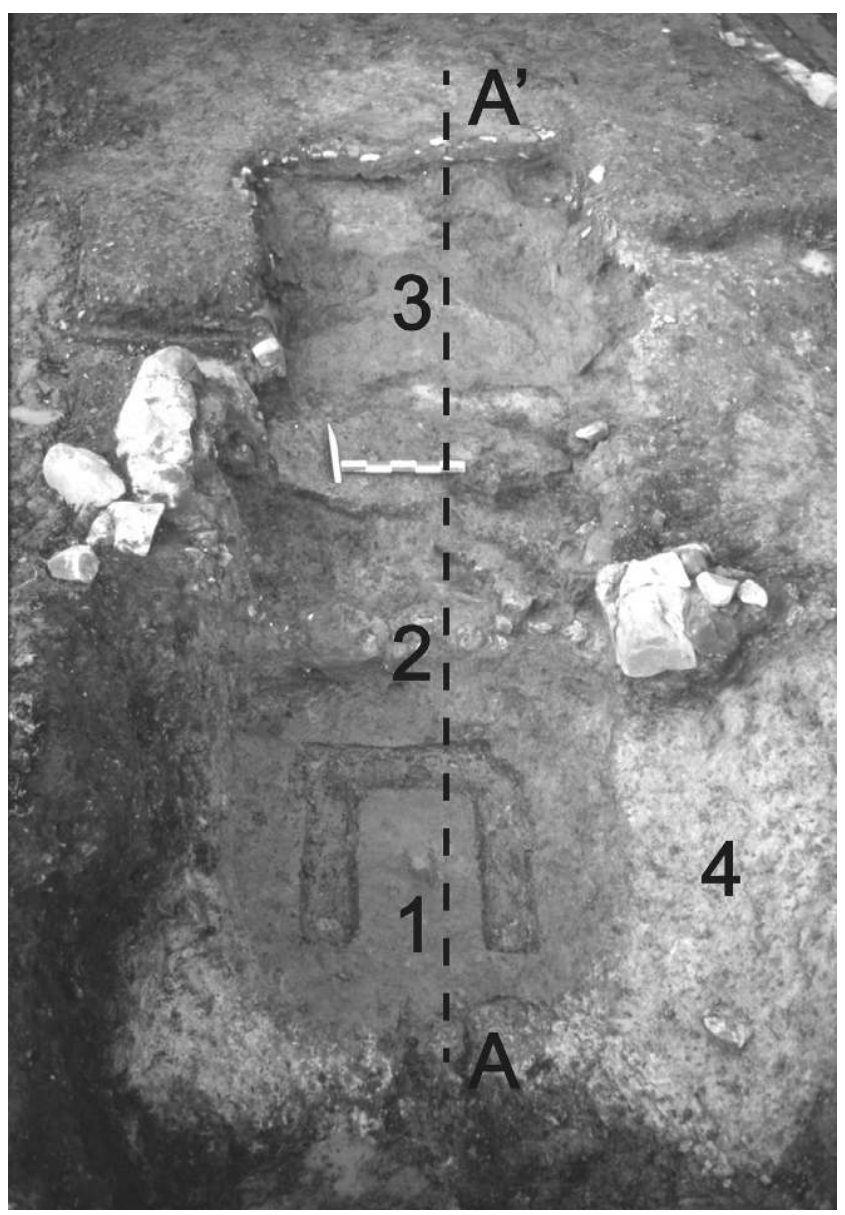

Figure 2 : (Voir planche couleur XIV) Le four à pots (A). 1) emprise du foyer bordé par un liseré de rubéfaction, avec traces des prélèvements archéomagnétiques. 2) marche qui sépare le foyer de la chambre de cuisson. 3) sole de la chambre. 4) argile en place dans laquelle le four a été excavé. A-A', coupe longitudinale (cf. figure 3).

Figure 2: (See colour plate XIV) Pottery kiln 'A). 1) position of the hearth outlined by rubefaction, with traces left by the archaeomagnetic sampling. 2) step separating the hearth and the firing chamber. 3) floor of the firing chamber. 4) natural clay into which the kiln had been constructed. A-A', longitudinal section (cf. fig. 3).

Le premier (four $\mathrm{A}$, figure 2), est un four à pots longitudinal mesurant près de $5 \mathrm{~m}$ de longueur et $1,30 \mathrm{~m}$ de largeur, dont la chambre de cuisson devait avoir tout au plus 2 à $3 \mathrm{~m}^{3}$ de capacité. C'est un des rares fours médiévaux longitudinaux dont l'intégralité du plan au sol est, semble-t-il, préservée ${ }^{4}$. Ses caractères morphologiques et dimensionnels

4. On s'en convaincra en parcourant la bibliographie sur le sujet, notamment Bucur et al., 1984; Chapelot et al., 1987; Bucur et al., 1989; sont proches de ceux du four à pots d'un atelier médiéval voisin dont l'abandon pourrait dater de la seconde moitié

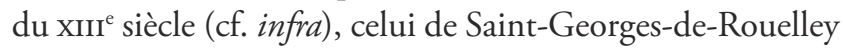
(Bucur et al., 1989, 228), comme le suggèrent le profil du foyer ou le pendage de la sole de la chambre de cuisson (figure 3). Les rebuts de fabrication associés au four A de la Goulande sont également analogues à ceux qui proviennent de l'atelier de Saint-Georges (Bucur et al., 1989). En effet, on trouve dans les deux ateliers les mêmes oules, jattes, cruches, vases à œil-de-perdrix (figure 4), etc., (Bucur et al., 1989) fabriqués à partir du même type de matière première argileuse.

À environ $1,50 \mathrm{~m}$ à l'est du four à pots, se trouvent les restes d'un four à tuiles (B), transformé en four à pots (C) (figure $5 \mathrm{a}$ et $5 \mathrm{~b}$ ). Le four à tuiles, conservé sur une cinquan-

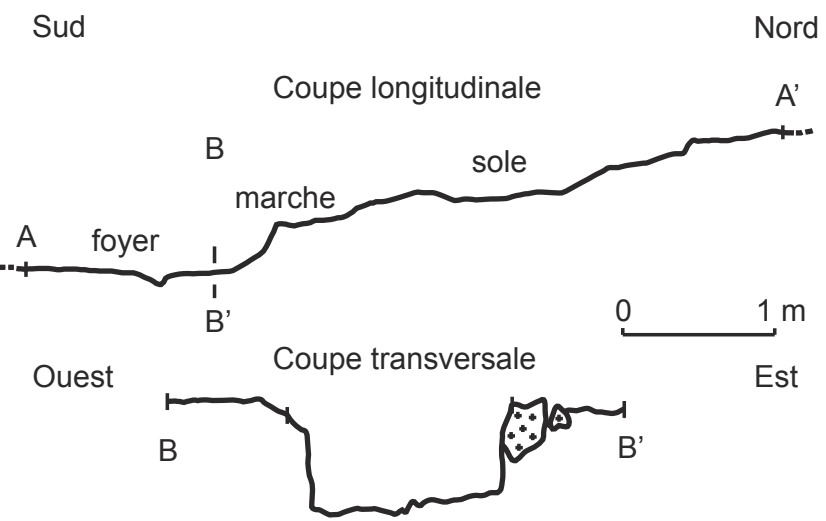

Figure 3: Coupes longitudinale et transversale du four A (cf. fig. 2). Figure 3: Longitudinal and transversal sections of kiln A (cf. fig. 2).

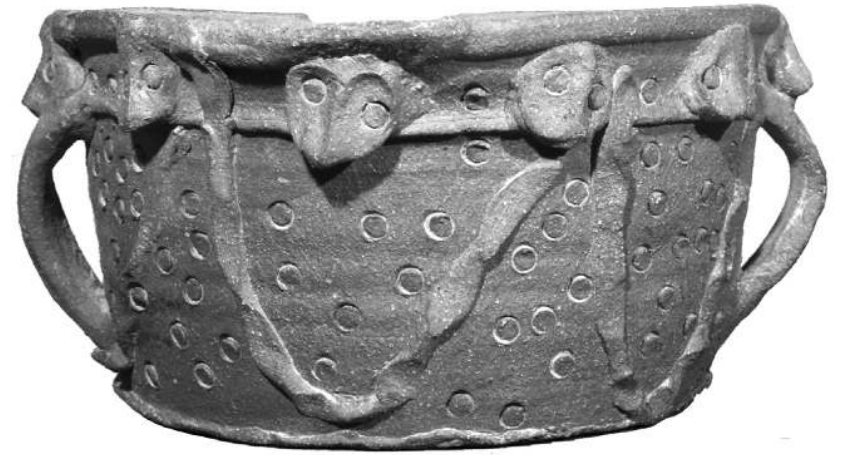

Figure 4 : Bassin à œil-de-perdrix (GLD 93-23), production du four A. $\mathrm{H}=17,4 \mathrm{~cm} ; \mathrm{L}=36,5 \mathrm{~cm}$.

Figure 4: "Eil-de-perdrix" basin (GLD 93-23), production of kiln A. $H=17,4 \mathrm{~cm} ; D=36,5 \mathrm{~cm}$.

Cartier et Durand, 1990; Guadagnin, 2000; Flambard-Héricher, 2002; Heege, 2007; Thuilier et al., 2015. 


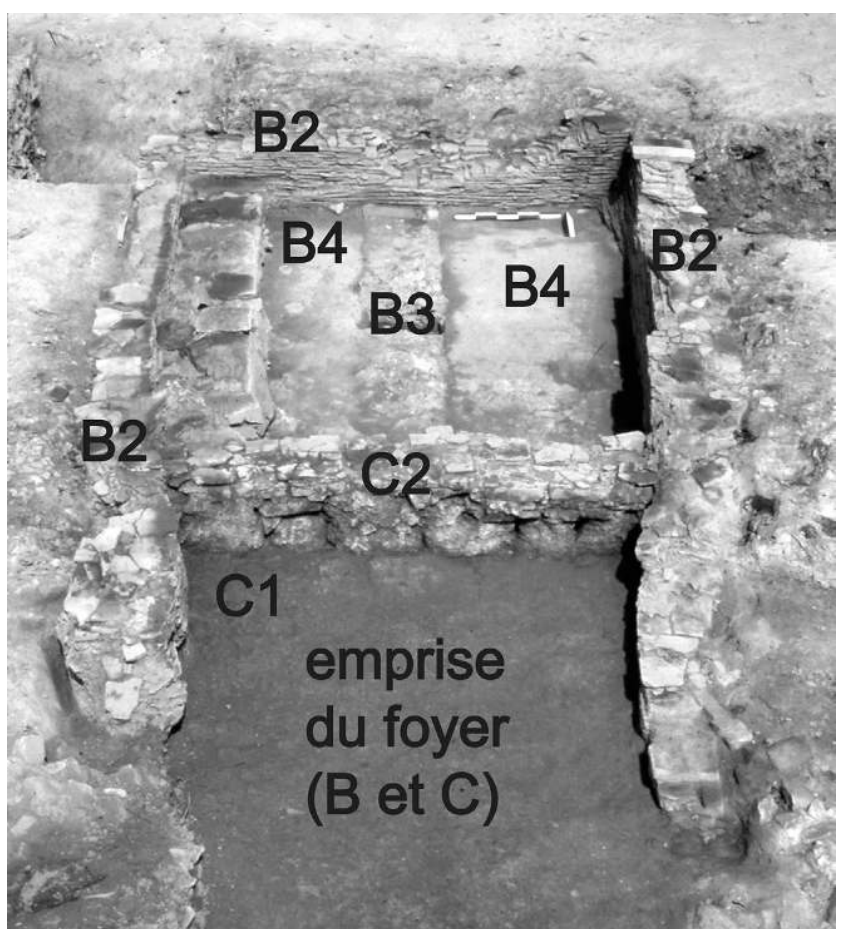

Figure 5a : (Voir planche couleur XIV) Le four à tuiles (B) et les restes du four à pots $(\mathrm{C})$.

Figure 5a: (See colour plate XIV) Tile kiln (B) and the remains of a pottery kiln $(C)$.

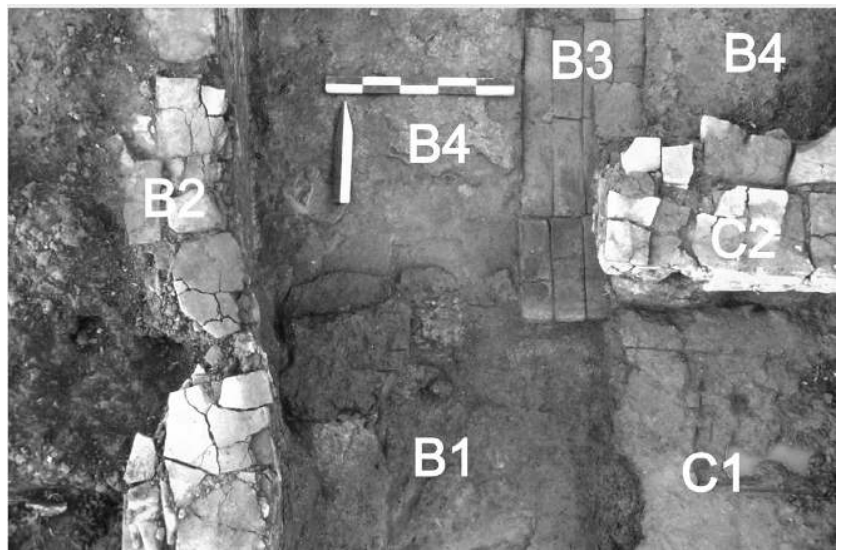

Figure 5b : (Voir planche couleur XIV) Le foyer très induré du four à tuiles (B1) en continuité avec les couloirs de chauffe (B4), sous $\mathrm{C} 1$ et $\mathrm{C} 2$.

Figure 5b: (See colour plate XIV) Hardened hearth floor of tile kiln (B1), firing tunnels (B4), under C1 and C2.

taine de $\mathrm{cm}$ de hauteur, mesure environ $5 \mathrm{~m}$ de longueur et environ 2,5 m de largeur. Il est caractérisé par des murs de tuileaux (B2) formant un plan rectangulaire ouvert sur le foyer, au sud. À égale distance des parois (B2), on trouve la trace au sol d'une languette centrale (B3), séparant deux

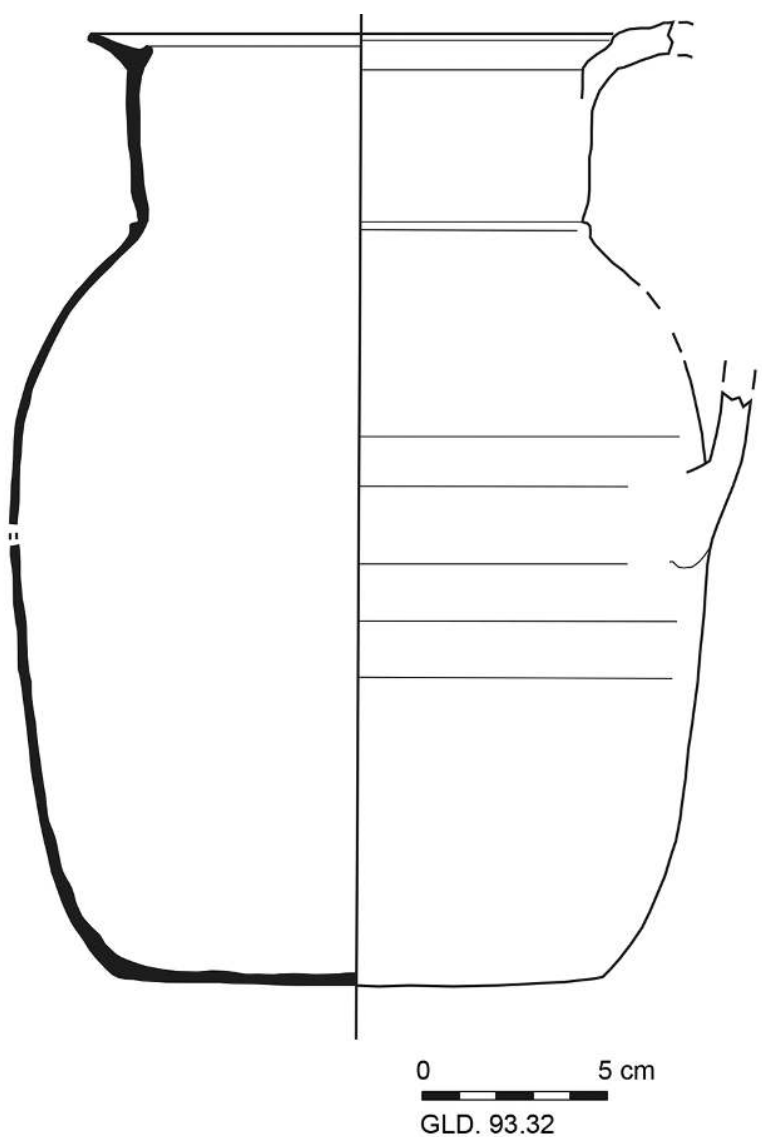

Figure 5c : Grès à pâte fine (GLD 93-32), lié au four C. H $=25,3 \mathrm{~cm}$; diamètre $=18,6 \mathrm{~cm}$.

Figure 5c: Fine fabric stoneware (GLD 93-32), associated with pottery kiln $(C), H=25,3 \mathrm{~cm}$; diameter $=18,6 \mathrm{~cm}$.

couloirs de chauffe, dans la continuité spatiale d'un premier foyer (B1, sous le niveau C1, figure 5b). Sur cette languette reposait la partie centrale de la sole perforée du four à tuiles. Après l'abandon de l'activité tuilière et la destruction de la languette centrale, au niveau de l'entrée des couloirs de chauffe, un muret de pierres haut d'une vingtaine de $\mathrm{cm}$ de hauteur (C2), recouvert d'une couche de tuileaux, a été aménagé entre les parois, en guise de marche séparant le foyer de la chambre de cuisson. Un second sol de foyer (sol de chauffe, $\mathrm{C} 1$ ), d'une dizaine de $\mathrm{cm}$ d'épaisseur et très induré, est venu recouvrir le sol de foyer (sol de chauffe, B1, très induré également) du four à tuiles en venant buter contre la marche qui porte elle-même les traces de fortes chauffes. Dans cet espace $(\mathrm{C} 1)$ et au-delà de la marche $(\mathrm{C} 2)$, ont été retrouvés des rebuts de grès à pâte fine (figure $5 \mathrm{c}$ ), du type de ceux que l'on ne rencontre pas avant la fin du Moyen Âge, mêlés à de la terre rubéfiée. Ces éléments semblent indiquer qu'il s'agit de céramiques utilisées, au moins en partie, dans le bâti (voûtement?) du four à pots. 


\section{Les Fours De LA Goulande : RÉSULTATS ARCHÉOMAGNÉTIQUES}

Les directions archéomagnétiques sont différentes les unes des autres, ce qui montre des âges différents pour la dernière utilisation de ces trois fours (figure 6). Nous avons effectué des datations archéomagnétiques en utilisant une nouvelle version de la courbe de référence des variations directionnelles du champ magnétique terrestre en France au travers des deux derniers millénaires (Le Goff et al., en préparation; trait bleu épais sur la figure 6 , à comparer au double trait rose retraçant la courbe établie précédemment par I. Bucur en 1994). Rappelons que cette courbe de référence est construite à l'aide de fenêtres glissantes de durée variable suivant la distribution temporelle des données archéomagnétiques datées disponibles (Le Goff et al., 2002). La méthode de datation consiste à comparer la distance angulaire $\gamma$ entre chacune des directions à dater et l'ensemble des directions moyennes datées définissant la courbe de référence à une distance angulaire critique $\gamma c$ en dessous de laquelle les deux directions comparées sont considérées identiques à $95 \%$ (Le Goff et al., 2002). Une datation archéomagnétique présentant $95 \%$ de certitude est donc obtenue quand $\gamma-\gamma c$ est inférieur à 0 (trait bleu et losanges blancs, figure 7). Le paramètre $\mathrm{p}$ (trait vert sur la figure 7) permet de définir un âge plus probable à l'intérieur de l'intervalle d'âge à $95 \%$ de certitude.

Nous avons obtenu trois datations archéomagnétiques avec $95 \%$ de certitude : pour le four A entre [1245 et 1315] (sans possibilité de proposer un âge plus probable à l'intérieur de cette fourchette, $\mathrm{p}$ étant trop faible); pour le four B, entre [1360 et 1465], avec un âge plus probable vers 14101420 et, pour le four C, une datation entre [1415 et 1530], avec un âge plus probable vers 1470-1480.

\section{Déclinaison}

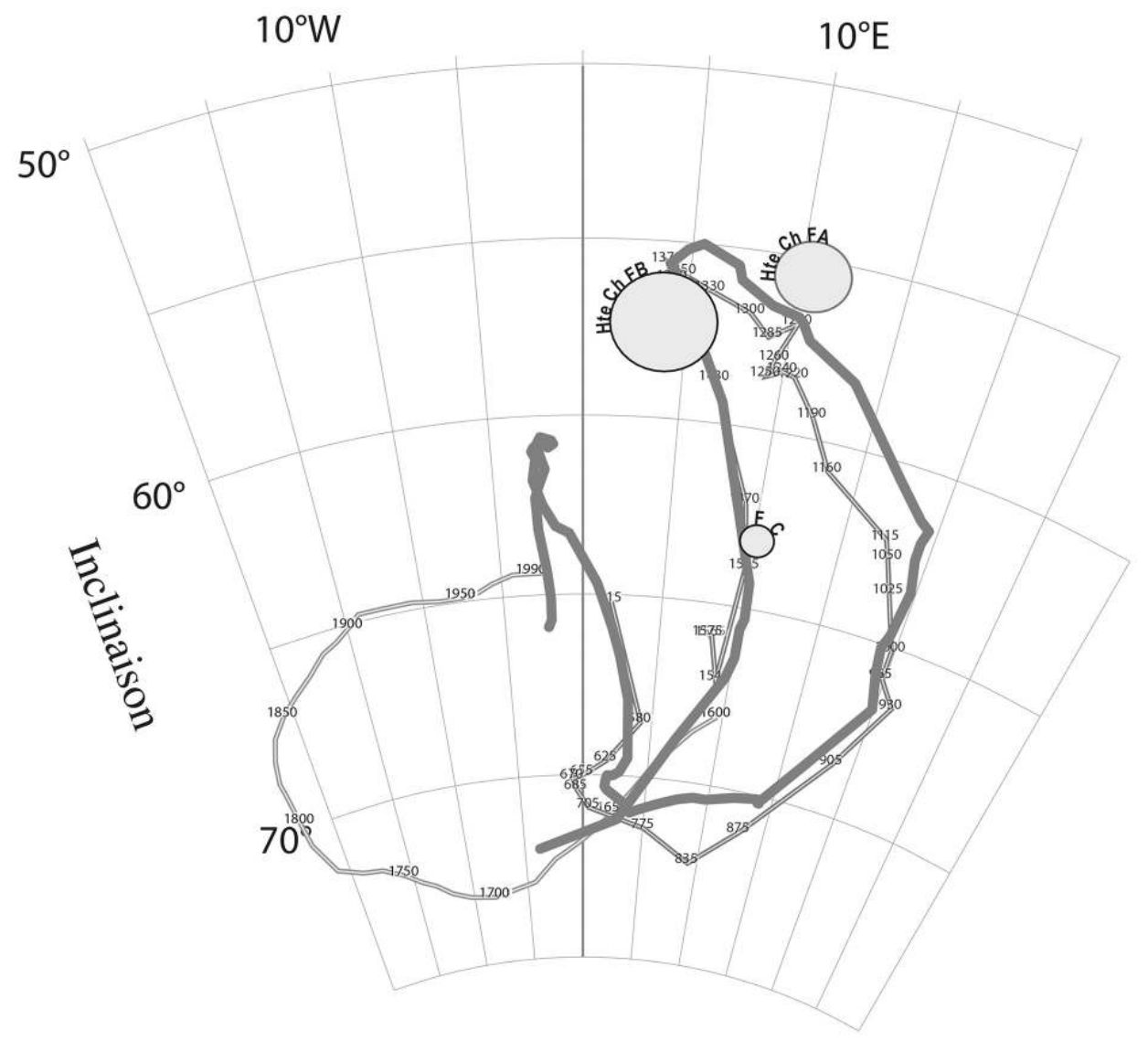

Figure 6: (Voir planche couleur XV) Les fours A, B et C dans la courbe de référence des variations directionnelles du champ magnétique terrestre en France. Figure 6: (See colour plate XV) Kilns $A, B$ and $C$ within the reference curve indicating the directional variations of the earth's magnetic field in France. 

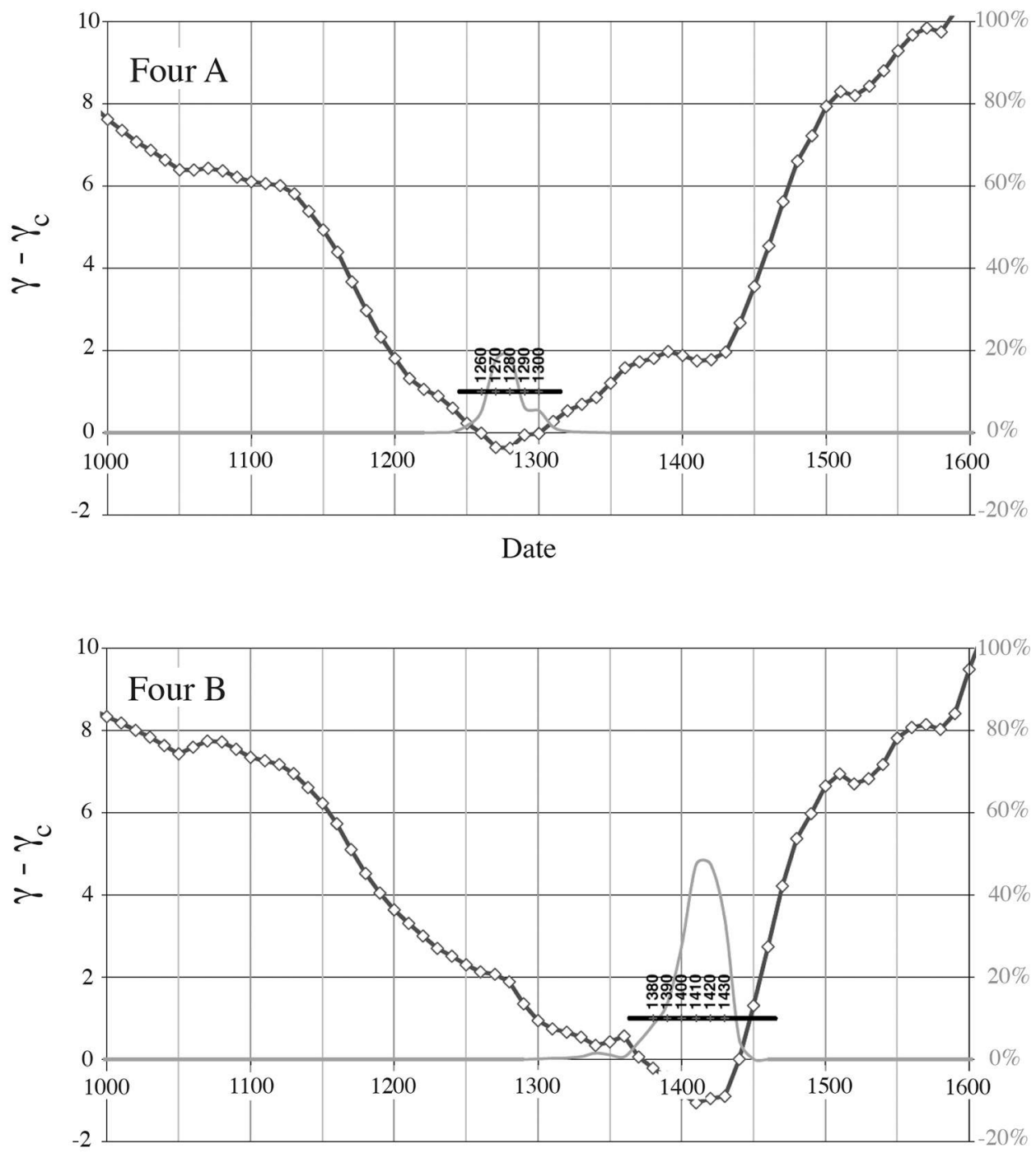

Date

Figure 7 : Résultats archéomagnétiques obtenus sur les fours A, B, C de l'atelier de la Goulande. Figure 7: Archeomagnetic results obtained on the kilns $A, B, C$ of the Goulande workshop.

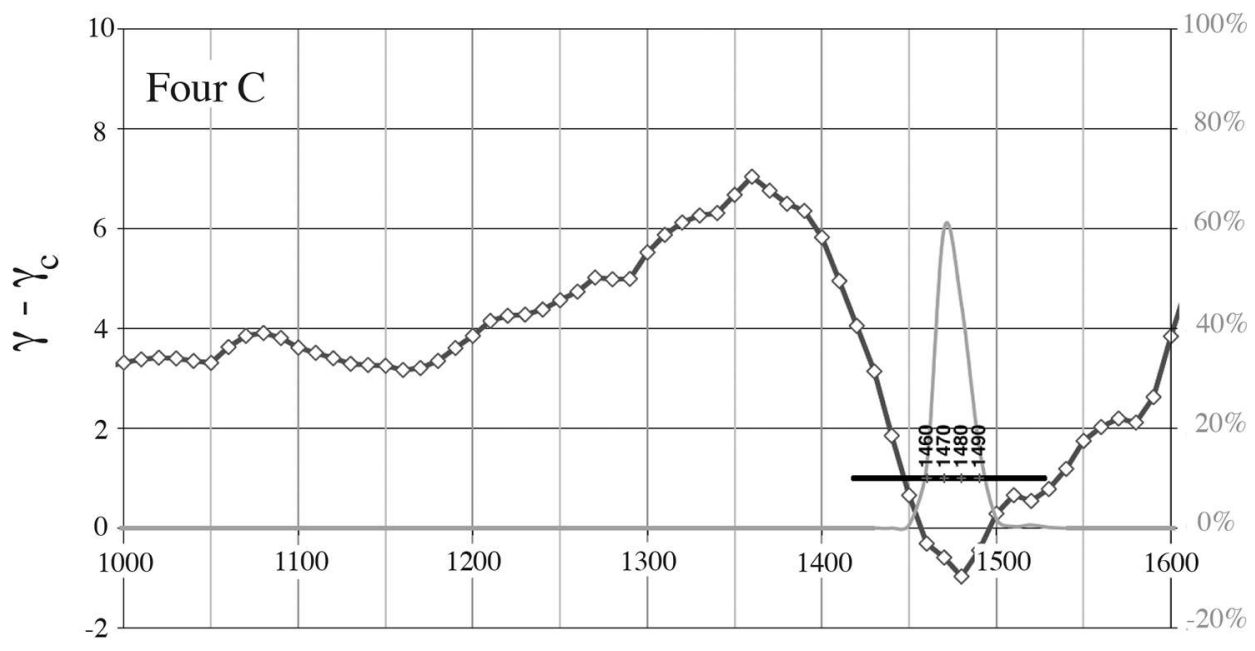

Date

ArcheoSciences, revue d'archéométrie, 41(2), 2017, p. 53-61 
Saint-Georges-de-Rouelley

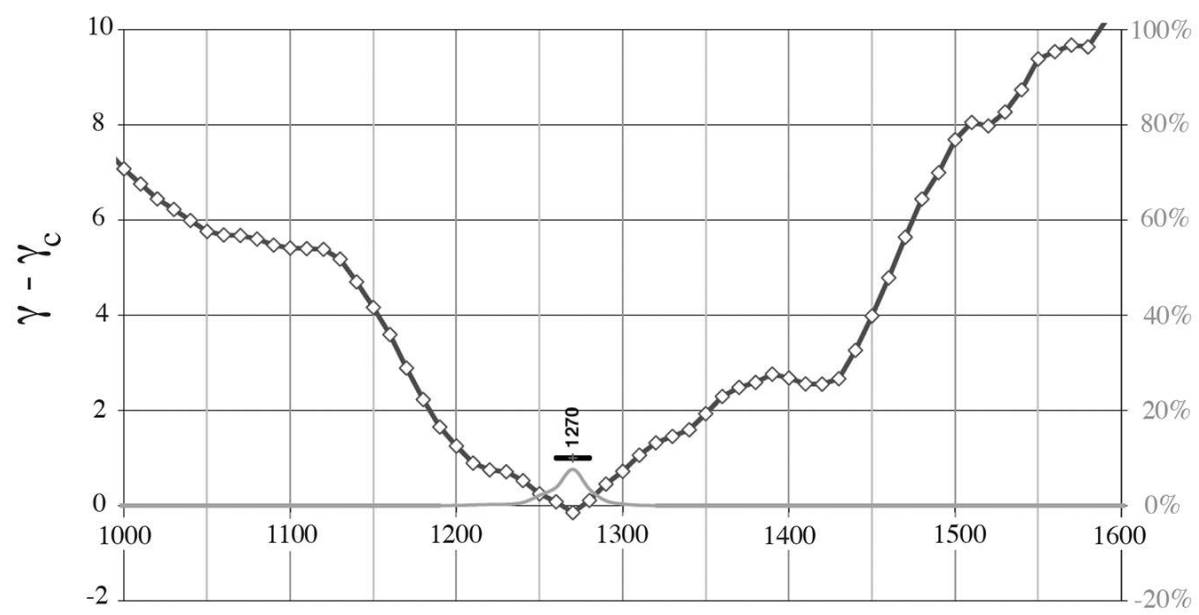

Figure 8 : Nouvelle datation archéomagnétique de l'atelier de potier de Saint-Georges-de-Rouelley (Manche).

Figure 8: New archeomagnetic dating of the pottery workshop in SaintGeorges-de-Rouelley (Manche).

Date

\section{Conclusions}

En accord avec les données archéologiques disponibles, les résultats archéomagnétiques donnent donc des datations différentes pour les trois fours A, B et C. L'atelier de la Goulande est un établissement pérenne, dont l'activité s'étale sur au moins trois siècles, même si elle a pu connaître des interruptions qu'il est difficile d'appréhender (pendant la guerre de Cent Ans?). Au XIII ${ }^{e}$ siècle, et peut-être avant, le four A produisait les mêmes types céramiques que l'atelier voisin de Saint-Georges-de-Rouelley, dont il aurait pu même être le concurrent, comme le montre la nouvelle datation archéomagnétique (1260-1280) du four de Saint-Georges (cf. infra), qui correspond mieux à ce que les données archéologiques et la typologie céramique laissaient entrevoir avant cela. La production de tuiles du four B, qui s'est développée ensuite, pourrait avoir pris fin au début du Xve siècle. Finalement, la production de grès du four $\mathrm{C}$ s'est substituée, sans doute pendant peu de temps, à la production de tuiles, dans le courant du $\mathrm{Xv}^{\mathrm{e}}$ siècle, pour une cessation définitive au plus tard au début du Xvi ${ }^{\mathrm{e}}$ siècle.

\section{Une nouvelle datation archéomagnétique de l'atelier de potier médiéval de Saint-Georges- de-Rouelley (Manche)}

En 1984, à l'occasion de travaux de terrassement liés à la création d'un lotissement, des tessonnières et les vestiges d'un four à pots ont été découverts dans le bourg de SaintGeorges-de-Rouelley, environ $6 \mathrm{~km}$ à l'ouest de la Goulande, au lieu-dit «La Potterie " (Bucur et al., 1989). Le four, de type longitudinal, amputé d'une partie de la chambre de cuisson, était conservé sur environ 3,30 m de longueur, dont environ 1,30 m de foyer. Au niveau de la marche qui le séparait de la chambre de cuisson, le four mesurait environ $1,20 \mathrm{~m}$ de largeur. Ses caractères morphologiques et dimensionnels sont donc proches de ceux du four A de la Goulande (cf. supra). Les productions céramiques de l'atelier de Saint-Georges-de-Rouelley sont elles aussi très voisines, $\mathrm{du}$ point de vue typologique, des premières productions de la Goulande. En effet, on retrouve dans les deux sites, fabriquées avec le même type de pâte argileuse, les mêmes formes céramiques : oules, cruches, pichets, vases à œil-deperdrix, etc.

Compte tenu de l'état d'élaboration de la courbe de référence des variations du champ magnétique terrestre au milieu des années 1980, Iléana Bucur avait retenu la période 1325-1350 pour l'abandon du four à pots. Une nouvelle datation archéomagnétique a été obtenue en utilisant la méthode de Le Goff et al. (2002) et la nouvelle courbe de référence des variations directionnelles en France depuis 2000 ans. Nous trouvons une datation archéomagnétique à $95 \%$ de certitude entre [1260 et 1280] (fig. 8). À noter que cette datation reste néanmoins difficile à établir, ce qui se traduit paradoxalement par un intervalle d'âge réduit ${ }^{5}$.

5. Cf. discussion dans Le Goff et al., 2002. 


\section{Bibliographie}

Bucur I., Dufournier D., Goulpeau L., Langouet L., Naveau J., 1984. La céramique à " œil-de-perdrix » et la production de la Hardelière à Laval (Mayenne). Archéologie médiévale, XIV, p. 169-219.

Bucur I., Dufournier D., Fajal B., Levalet D. et LouisPhilippe M., 1989. Un four de potier médiéval à Saint-Georges-de-Rouelley (Manche), les premiers grès basnormands. Archéologie Médiévale, XIX, p. 215-241.

Cartier J. et Durand M., 1990. Un four de potier médiéval à La Croix-Saint-Ouen (Oise), à la limite de la forêt de Compiègne. Revue archéologique de Picardie, n 3-4, p. 127-137.

Chapelot J., Galinié H., Pilet-Lemière J. (dir.), 2007. La céramique (Ve-XIXe siècle), Fabrication, Commercialisation, Utilisation, Actes du I ${ }^{\text {er }}$ Congrès international d'archéologie médiévale, Caen, 259 p.

Dufournier D. et Fajal B., 1995. L'apparition du grès dans la région domfrontaise, premières observations. In La céramique $d u X I^{e}$ au XVI siècle en Normandie, Beauvaisis, Ille-de-France, actes de la Table ronde du GRHIS, Rouen, p. 73-80.

FAJAL B., 1990. L'atelier de potier médiéval de La Goulande à La Haute-Chapelle (Orne). Sondage archéologique. Archéologie Médiévale (chronique des fouilles), XX, p. 448.

Fajal B., 1990. L'atelier de potier médiéval de La Potterie à Saint-Georges-de-Rouelley (Manche). "Fouille de sauvetage urgent ", Archéologie Médiévale (chronique des fouilles), XX, p. 450.

FajAL B., 1995. L'atelier producteur de grès médiéval de La Goulande à La Haute-Chapelle (Orne). Fouille program- mée. Archéologie Médiévale (chronique des fouilles), XXV, p. 344-345.

Fajal B, Marguerie D., avec la collaboration de Bucur I., et Bernouis P., 2015. L'atelier de potier médiéval de la Picaudière (La Haute-Chapelle, Orne) : four, soles et combustible. Archéosciences, no 39, p. 177-184 + pl. XVIII-XIX.

Flambard Héricher A.-M., 2002. Potiers et poteries du Bessin. Histoire et archéologie d'un artisanat rural du XI au XX' siècle en Normandie, Caen, Publications du CRAHAM, 407 p.

Guadagnin R., 2000. Fosses - Vallée de l'Ysieux. Mille ans de production céramique en Île-de-France. Volume 1. Les données archéologiques et historiques, Caen, Publications du CRAM, $367 \mathrm{p}$.

Heege A., 2007. Topferöfen. Die Erforschung frühmittelalterlicher bis neuzeitlicher Töpferöfen in Belgien, den Niederlanden, Deutschland, Österreich und der Schweiz. Basler Hefte zur Archäologie. Band 4. Archäologie-Verlag, Bâle, 432 p.

Lautridou J.-P., 2002. Les argiles fini-tertiaires de Saint-Gillesdes-Marais (Domfrontais, Orne). Bulletin de la société linéenne de Normandie, 118, p. 39-41.

Le Goff M., Gallet Y., Genevey A., et Warmé N., 2002. On archeomagnetic secular variation curves and archeomagnetic dating. Phys. earth. planet. inter, vol. 134, p. 203-211.

Mauger, 1905. Ger et ses poteries. In Annuaire de l'enseignement primaire de la Manche, t. 9, p. 209-220.

Thuilier F. et Louis É. (dir.), 2015. Tourner autour du pot... : les ateliers de potiers médiévaux du VI au XIII siècle dans l'espace européen, Actes du colloque international de Douai (5-8 octobre 2010), Caen, Presses universitaires de Caen, coll. " Publications du CRAHAM », 792 p. 